\title{
ROTA'S THEOREM FOR GENERAL FUNCTIONAL HILBERT SPACES
}

\author{
JOSEPH A. BALL
}

\begin{abstract}
By a theorem of G.-C. Rota, every (linear) operator $T$ on a Hilbert space with spectral radius less than one is similar to the adjoint of the unilateral shift $S$ of infinite multiplicity restricted to an invariant subspace. This theorem is shown to be true in a rather general context, where $S$ is multiplication by $z$ on a Hilbert space of functions analytic on an open subset $D$ of the complex plane, and $T$ is an operator with spectrum contained in $D$. A several-variable version for an $N$-tuple of commuting operators with a corollary concerning complete spectral sets is also presented.
\end{abstract}

1. Introduction. Some time ago, G.-C. Rota [11] showed that every (linear) Hilbert space operator $T$ with spectral radius less than 1 is similar to the restriction of the adjoint of the unilateral shift $S$ (of infinite multiplicity) to a suitable invariant subspace, and in this sense the unilateral shift is a "Universal Model" for such operators $T$. Later D. A. Herrero [10] and D. Voiculescu [14] obtained an analogous result with $T$ an operator with spectrum contained in a "nice" bounded open subset $D$ of the complex plane, and $S$ multiplication by $z$ on a certain Hilbert space of functions analytic on D. D.N.Clark [7] has obtained a several-variable version of the Rota theorem for $N$ commuting strict contractions. In this paper, by a relatively simple kernel function argument similar to a technique appearing in work of C. A. Berger and B. I. Shaw [3] and of R. G. Douglas and C. Pearcy [8], we generalize the above results as follows:

TheOREM 1. Let $D$ be a bounded open subset of the complex plane. Let $H$ be a Hilbert space of complex-valued functions on $D$ (the vector space operations being the usual point wise operations) such that

(1) each element $f$ in $H$ is analytic on $D$,

(2) for each $w$ in D, evaluation at $w(f \rightarrow f(w))$ is a bounded linear functional on $H$,

(3) the constant function 1 is in $H$, and

(4) the operator $S: f(z) \rightarrow z f(z)$ is a bounded operator mapping $H$ into itself.

For $K$ a Hilbert space, let $H_{K}=H \otimes K$ (the induced Hilbert space of $K$-valued functions), and let $S_{K}=S \otimes I_{K}$ on $H_{K}$. Then if $T$ is an operator on $K$

Received by the editors May 13, 1976 and, in revised form, September 13, 1976.

AMS (MOS) subject classifications (1970). Primary 47A20, 46E20.

Key words and phrases. Reproducing kernel function, Riesz-Dunford functional calculus, similarity, complete spectral set and normal dilation.

๑ American Mathematical Society 1977 
such that the spectrum $\sigma(T)$ of $T$ is contained in $D$, then the adjoint of $T$ is similar to the adjoint of $S_{K}$ restricted to a suitable invariant subspace.

To state the next theorem, we recall a definition. If $T=\left(T_{1}, \ldots, T_{N}\right)$ is an $N$-tuple of commuting operators on $K$, the joint spectrum $\sigma(T)$ of $T$ is defined to be the set of all $N$-tuples $w=\left(w_{1}, \ldots, w_{N}\right)$ in $e^{N}$ such that $p(w)$ belongs to the spectrum of $p(T)$ for every multivariable polynomial $p=$ $p\left(z_{1}, \ldots, z_{N}\right)[2]$.

TheOREM 2. Let $D=D_{1} \times \ldots \times D_{N}$ be a bounded polyopen subset of $\mathfrak{C}^{N}$ (i.e. each $D_{i}$ is open in $C_{, i}=1, \ldots, N$ ) and suppose $H$ is a Hilbert space of -valued functions on $D$ (the vector space operations the usual pointwise operations) such that

(1) each element $f$ in $H$ is analytic on $D$,

(2) for each $w=\left(w_{1}, \ldots, w_{N}\right)$ in $D$, evaluation at $w(f \rightarrow f(w))$ is a bounded linear functional on $H$,

(3) the constant function 1 is in $H$, and

(4) for $j=1, \ldots, N$, the operator $S_{j}: f\left(z_{1}, \ldots, z_{N}\right) \rightarrow z_{j} f\left(z_{1}, \ldots, z_{N}\right)$ is a bounded operator mapping $H$ into itself.

For $K$ a Hilbert space, let $H_{K}=H \otimes K$ and $S_{K, j}=S_{j} \otimes I_{K}$ on $H_{K}$ $(j=1, \ldots, N)$. Then if $T=\left(T_{1}, \ldots, T_{N}\right)$ is an $N$-tuple of commuting operators on $K$ such that $\sigma(T) \subset D$, then $T$ is similar to the adjoint of $S_{K}=$ $\left(S_{K, 1}, \ldots, S_{K, N}\right)$ restricted to a suitable invariant subspace; more precisely, there is a bounded invertible map $\Gamma$ from $K$ onto a subspace of $H_{K}$ invariant under $S_{K_{j}}^{*}$ for each $j$, such that $S_{K_{j}}^{*} \Gamma=\Gamma T_{j}^{*}$.

2. Proof of Theorem 1. Let $D, H$ and $K$ be as in the hypotheses of Theorem 1. By (2) and the Riesz-Fréchet theorem there is a reproducing kernel function $k(\bar{w}, z)$, which, for each $w$ in $D$, belongs to $H$ as a function of $z$, and has the reproducing property

$$
\langle f, k(\bar{w}, \cdot)\rangle_{H}=f(w) .
$$

The computation, for $f$ in $H$ and $w$ in $D$,

$$
\left\langle f, S^{*} k(\bar{w}, \cdot)\right\rangle_{H}=\langle S f, k(\bar{w}, \cdot)\rangle_{H}=w f(w)=\langle f, \bar{w} k(\bar{w}, \cdot)\rangle_{H}
$$

shows

$$
S^{*} k(w, \cdot)=w k(w, \cdot) \text { for } w \text { in } D^{*}(=\{z: \bar{z} \in D\}) .
$$

Since $k(w, z)=\langle k(w, \cdot), k(\bar{z}, \cdot)\rangle_{H}=\langle\overline{k(\bar{z}, \cdot), k(w, \cdot)}\rangle_{H}=\overline{k(\bar{z}, \bar{w})}$, hypothesis (1) implies

(c) for each $z$ in $d, k(w, z)$ is analytic on $D^{*}$ as a function of $w$.

Since $\sigma(T)$ is a compact subset of the open set $D$, there is a curve $C$ contained in $D$, consisting of a finite number of rectifiable Jordan curves, and containing $\sigma(T)$ in its interior. Then $C^{*}$ (the image of $C$ under complex conjugation) is a curve contained in $D^{*}$ and containing $\sigma\left(T^{*}\right)$ in its interior. By (c) we can define via the Riesz-Dunford functional calculus [7, VII.3], for each $x$ in $K$, a $K$-valued function of $z,(\Gamma x)(z)$ by 


$$
\begin{aligned}
(\Gamma x)(z) & =k\left(T^{*}, z\right) x=\left\{\int_{C^{*}} k(w, z)\left(w I-T^{*}\right)^{-1} d w\right\} x \\
& =\int_{C^{*}} k(w, z)\left(w I-T^{*}\right)^{-1} x d w .
\end{aligned}
$$

Since $\|k(w, \cdot)\|_{H}^{2}=k(w, \bar{w})$ is continuous on $D^{*}$, a compactness argument gives $\sup _{w \in C^{*}}\|k(w, \cdot)\|_{H}<\infty$. Similarly $\sup _{w \in C^{*}}\left\|\left(w I-T^{*}\right)^{-1}\right\|<\infty$, and hence,

$$
\|\Gamma x\|_{H_{K}} \leqslant \sup _{w \in C^{*}}\|k(w, \cdot)\|_{H} \sup _{w \in C^{*}}\left\|\left(w I-T^{*}\right)^{-1}\right\| l\left(C^{*}\right)\|x\| \cdot
$$

Thus $\Gamma$ is a bounded map of $K$ into $H_{K}$.

Next we show $\Gamma$ is bounded below. By hypothesis (3), the constant function $1 \in H$; let $\alpha=\|1\|_{H}>0$. For $x$ in $K, x \equiv 1 \otimes x$ is an element of $H_{K}$ with $\|x\|_{H_{K}}=\alpha\|x\|_{K}$. Then

$$
\begin{aligned}
\langle x, \Gamma x\rangle_{H_{K}} & =\left\langle x, \int_{C^{*}} k(w, \cdot)\left(w I-T^{*}\right)^{-1} x d w\right\rangle_{H_{K}} \\
& =\int_{C}\left\langle(\bar{w} I-T)^{-1} x, k(w, \cdot) x\right\rangle_{H_{K}} d \bar{w} \\
& =\int_{C}\left\langle(\bar{w} I-T)^{-1} x, x\right\rangle_{K} d \bar{w} \quad \text { by (a) } \\
& =\langle x, x\rangle_{K} \quad \text { by the functional calculus for } T .
\end{aligned}
$$

Hence

$$
\|x\|_{K}^{2}=\langle x, \Gamma x\rangle_{H_{K}} \leqslant\|x\|_{H_{K}}\|\Gamma x\|_{H_{K}}=\alpha\|x\|_{K}\|\Gamma x\|_{H_{K}}
$$

or $\|\Gamma x\|_{H_{K}} \geqslant \alpha^{-1}\|x\|_{K}$, and hence $\Gamma$ is bounded below.

Finally, we compute

$$
\begin{aligned}
\Gamma T^{*} x & =\int_{C^{*}} k(w, \cdot)\left(w I-T^{*}\right)^{-1}\left(T^{*} x\right) d w \\
& =\int_{C^{*}} w k(w, \cdot)\left(w I-T^{*}\right)^{-1} x d w-\int_{C^{*}} k(w, \cdot) x d w \\
& =\int_{C^{*}} w k(w, \cdot)\left(w I-T^{*}\right)^{-1} x d w \quad \text { by (c) } \\
& =\int_{C^{*}}\left(S^{*} k(w, \cdot)\right)\left(w I-T^{*}\right)^{-1} x d w \quad \text { by (b) } \\
& =S_{K}^{*} \Gamma x
\end{aligned}
$$

Hence $\operatorname{Ran} \Gamma$ is invariant under $S_{K}^{*}$, and $T^{*}$ is similar to $S_{K}^{*} \mid \operatorname{Ran} \Gamma$ via $\Gamma$.

3. Proof of Theorem 2. Let $D, H, K, T=\left(T_{1}, \ldots, T_{N}\right)$ and $S=$ $\left(S_{1}, \ldots, S_{N}\right)$ be as in the statement of Theorem 2. Just as before, for each $w=\left(w_{1}, \ldots, w_{N}\right)$ in $D$, there is a reproducing kernel function $k(\bar{w}, z)(\bar{w}=$ $\left.\left(\bar{w}_{1}, \ldots, \bar{w}_{N}\right)\right)$ which belongs to $H$ as a function of $z$ and satisfies

(a) for $f$ in $H, w$ in $D,\langle f, k(\bar{w}, \cdot)\rangle_{H}=f(w)$, 
(b) $S_{j}^{*} k(w, \cdot)=w_{j} k(w, \cdot)$ for $w$ in $D^{*}$, and

(c) for each $z$ in $D, k(w, z)$ is analytic on $D^{*}$ as a function of $w$.

For $\lambda=\left(\lambda_{1}, \ldots, \lambda_{N}\right)$ in $e^{N}$, let $\pi_{i}: \lambda \rightarrow \lambda_{i}$ be the projection onto the $i$ th coordinate. An elementary argument $\left[2\right.$, p. 275] shows that $\pi_{i}(\sigma(T))=\sigma\left(T_{i}\right)$. Since $D=D_{1} \times \cdots \times D_{N}$ is polyopen and $\sigma(T) \subset D$, it follows that $\sigma\left(T_{i}\right)$ $\subset D_{i}$. Let $C_{i}$ be a curve contained in $D_{i}$ consisting of finitely many rectifiable Jordan curves and containing $\sigma\left(T_{i}\right)$ in its interior. Define $\Gamma: K \rightarrow H_{K}$ by

$$
\Gamma x=\int_{C_{N}^{*}} \ldots \int_{C_{1}^{*}} k(w, \cdot)\left(w_{N} I-T_{N}^{*}\right)^{-1} \ldots\left(w_{1} I-T_{1}^{*}\right)^{-1} x d w_{1} \ldots d w_{N}
$$

The proof that $\Gamma$ is bounded, bounded below, and that $\Gamma T_{i}^{*}=S_{K, i}^{*} \Gamma(i=$ $1, \ldots, N)$ now proceeds exactly as in the proof of Theorem 1 .

4. Examples. In this section we collect several examples of open sets $D$ and corresponding spaces $H$ satisfying conditions (1)-(4) of Theorem 1 or of Theorem 2.

ExAmple 1. Let $D$ be a bounded domain of $C$ and let $H$ be the space of single-valued functions $f$ analytic on $D$ with modulus square-integrable with respect to area measure: $\iint_{D}|f(z)|^{2} d x d y<\infty$. The verification of hypothesis (2) follows by an argument involving Taylor series [4, p. 5]; the others follow easily. For the case where $D$ is the unit disk, the associated operator $S: f(z)$ $\rightarrow z f(z)$ is the Bergman shift, and the kernel function $k(\bar{w}, z)$ is given by $\pi^{-1}(1-\bar{w} z)^{-2}$.

ExAmple 2. Let $D$ be a bounded domain in $\bigodot$ whose boundary consists of a finite number of nonintersecting, analytic Jordan curves and let $\mu$ be any measure supported on $\partial D$ which is boundedly mutually absolutely continuous with respect to arc-length measure on $\partial D$. Let $H$ be the closure of $\operatorname{Rat}(\bar{D})$ (rational functions with poles off of $\bar{D}$ ) in the space $L^{2}(\mu)$. An application of the Cauchy integral formula enables one to verify (1) and (2) for $(D, H)[4$, pp. 77-78], and (3) and (4) follow easily. This is essentially the situation considered by Herrero [10]. The corresponding operator $S$, for special choices of $(D, H)$, has been studied by Sarason [12] and Abrahamse and Douglas [1]. When $D$ is the unit disk and $\mu$ is arc-length measure on $\partial D, S$ is the unilateral shift and the kernel function $k(\bar{w}, z)$ is equal to $(1-\bar{w} z)^{-1}$. In this case the map $\Gamma$ in the proof of Theorem 1 specializes to that originally used by Rota [11].

The above construction works equally well with $H$ chosen to be the closure of the polynomials in $L^{2}(\mu)$.

EXAMPLE 3. Let $D$ be arbitrary bounded domain in $C$. We show that there is a measure $\mu$ with support contained in $\partial \bar{D}$, such that, if $H$ is the closure of $\operatorname{Rat}(\bar{D})$ in $L^{2}(\mu),(D, H)$ satisfies (1)-(4). The argument again works equally with $H$ chosen to be the closure of the ploynomials in $L^{2}(\mu)$. The techniques are adapted from the work of Brennan [6].

Let $t$ be some fixed point in $D$. By the maximum modulus theorem, evaluation at $t$ is a bounded linear functional on $\operatorname{Rat}(\bar{D})$ as a subspace of 
$C(\partial \bar{D})$ (continuous functions on $\partial \bar{D}$ with sup norm), and hence, by the Hahn-Banach theorem and Riesz representation theorem, there is a measure $\mu$ supported on $\partial \bar{D}$ such that

$$
f(t)=\int f(z) d \mu(z) \text { for all } f \text { in } \operatorname{Rat}(\bar{D}) .
$$

Let $H$ be the closure of $\operatorname{Rat}(\bar{D})$ in $L^{2}(\mu)$-norm, and let us say, for $w$ a point in $D$, " $w$ is a bounded evaluation" if evaluation at $w$ is a bounded linear functional on $\operatorname{Rat}(\bar{D})$ in $L^{2}(\mu)$-norm.

Since $|f(t)| \leqslant \int|f(z)| d \mu(z) \leqslant\|f\|_{L^{2}(\mu)}$ for $f$ in $\operatorname{Rat}(\bar{D}), t$ is a bounded evaluation. The operator $S$ of multiplication by $z$ on $H$ is subnormal with minimal normal extension $N$ equal to multiplication by $z$ on $L^{2}(\mu)$, which has spectrum $\sigma(N) \subseteq \partial \bar{D}$. Since $(S-t I) H$ is in the kernel of the nontrivial bounded linear functional $f \rightarrow f(t)$ on $H, S-t I$ is not onto, and hence $t \in \sigma(S)$. By a result of Bram [5, Theorem 4], it follows that $D \subset \sigma(S)$.

Let $w \in D$ and suppose $w$ is not a bounded evaluation. Let $g$ be any function in $L^{2}(\mu)$ such that $\int f g d \mu=0$ for all $f$ in $H$. Then, in particular, for $f$ in $\operatorname{Rat}(\bar{D}),(f(z)-f(w)) /(z-w)$ is also in $\operatorname{Rat}(\bar{D})$, and hence

$$
\int \frac{f(z)-f(w)}{z-w} g(z) d \mu(z)=0,
$$

or

$$
\int f(z) \frac{g(z)}{z-w} d \mu(z)=f(w) \int \frac{g(z)}{z-w} d \mu(z) .
$$

Since $g(z)(z-w)^{-1} \in L^{2}(\mu)$ ( $w$ is away from supp $\mu$ ) and $w$ is not a bounded evaluation, it follows that $\int[g(z) /(z-w)] d \mu(z)=0$. Hence

$$
(z-w)^{-1} \in H .
$$

But this implies that multiplication by $(z-w)^{-1}$ is a bounded inverse for $(S-w)$ on $H$, contradicting that $w \in \sigma(S)$. Hence to each point $w$ in $D$ there corresponds a bounded linear functional $\lambda_{w}$ on $H$ such that $f(w)=$ $\lambda_{w}(f)$ for all $f$ in $\operatorname{Rat}(\bar{D})$.

To show that $f(w) \equiv \lambda_{w}(f)$ is analytic on $D$ for each $f$ in $H$, it suffices to show (via a normal family argument) that $\left\|\lambda_{w}\right\|$ is uniformly bounded on compact subsets of $D$. This will follow if we show that, for any $w_{0}$ in $D,\left\|\lambda_{w}\right\|$ is uniformly bounded for $w$ in some neighborhood of $w_{0}$. For $w_{0}$ in $D$, an argument above gives $\left(z-w_{0}\right)^{-1} \notin H$. Hence there exists a $g \in L^{2}(\mu)$ such that $\int f g d \mu=0$ for all $f$ in $H$ but $\int\left(z-w_{0}\right)^{-1} g(z) d \mu(z)=1$. Then for $f$ in $\operatorname{Rat}(\bar{D}), w$ in $D,(*)$ holds. But for $w$ sufficiently close to $w_{0}$,

$$
\left|\int(z-w)^{-1} g d \mu\right|>\frac{1}{2} \quad \text { and }\left\|(z-w)^{-1} g\right\|_{L^{2}(\mu)} \leqslant 2\left\|\left(z-w_{0}\right)^{-1} g\right\|_{L^{2}(\mu)} .
$$

Hence, (*) implies 


$$
\begin{aligned}
|f(w)| & =\left|\left\{\int(z-w)^{-1} g d \mu\right\}^{-1} \int(z-w)^{-1} g f d \mu\right| \\
& <2\left\|(z-w)^{-1} g\right\|_{L^{2}(\mu)}\|f\|_{L^{2}(\mu)} \leqslant 4\left\|\left(z-w_{0}\right)^{-1} g\right\|_{L^{2}(\mu)}\|f\|_{L^{2}(\mu)}
\end{aligned}
$$

Hence (1) and (2) follow for $(D, H)$. Properties (3) and (4) are trivial.

EXAMPLE 4. For $D$ a (not necessarily connected) bounded open set $\subseteq \mathcal{C}$, a space $H$ corresponding to $D$ can be constructed as follows. Let $\left\{D_{i}\right\}_{i=1}^{N}(N$ finite or $\infty$ ) be a listing of the components of $D$, and let $H_{i}$ be a space associated with $D_{i}$ as in any of the preceding examples. Let $H$ be the space of functions analytic on $D$ such that $f_{i} \equiv f \mid D_{i}$ is in $H_{i}$ for all $i$, with $\|f\|_{H}^{2}=$ $\Sigma\left\|f_{i}\right\|_{H_{i}}^{2} / 2^{i}\|1\|_{H_{i}}<\infty$. Then it is easily verified that $(D, H)$ satisfies (1)-(4).

In all of the above examples, the associated operator $S$ has been subnormal. Examples of spaces $(D, H)$ such that the associated $S$ is not subnormal can be obtained by considering the $H^{2}(\beta)$ and $L^{2}(\beta)$ spaces associated with a nonsubnormal weighted shift operator, as discussed by Shields [13].

Examples where $D$ is a polyopen subset of $e^{N}, N>1$, can be obtained by tensoring any combination of the above single-variable examples. The simplest example is obtained by taking $D$ equal to the unit polydisk, $\mu$ the measure on the distinguished boundary equal to the $N$-fold Cartesian product of linear Lebesgue measure on the unit circle and $H$ the closure of the polynomials in $L^{2}(\mu)$. In this case, the kernel function $k(\bar{w}, z)$ is equal to $\left(1-\bar{w}_{1} z_{2}\right)^{-1} \ldots\left(1-\bar{w}_{N} z_{N}\right)^{-1}$, and the similarity mapping $\Gamma$ in the proof of Theorem 2 is the same as that given by Clark [7].

5. Complete spectral sets. Recall, for $T=\left(T_{1}, \ldots, T_{N}\right)$, an $N$-tuple of commuting operators on a Hilbert space $K$, a compact set $X \subseteq \mathrm{e}^{N}$ is called a spectral set for $T$ if $X$ contains $\sigma(T)$ and $\|f(T)\| \leqslant \sup \{|f(z)|: z \in X\}$ for every $f$ in $\operatorname{Rat}(X)$. The related but stronger notion of complete spectral set is defined in [2], where Arveson shows that a necessary and sufficient condition for $X$ to be a complete spectral set for $T$ is that $\sigma(T) \subseteq X$ and that $T$ have a normal dilation $N=\left(N_{1}, \ldots, N_{N}\right)$ (i.e., the $N_{i}$ 's are commuting normal operators defined on a larger space $K^{\prime}$ such that $r(T)=P_{K} r(N) \mid K$ for all $r \in \operatorname{Rat}(X))$ with $\sigma(N) \subseteq \partial X$. Using this result and Theorem 2, we obtain

Corollary. Let $T=\left(T_{1}, \ldots, T_{N}\right)$ be an $N$-tuple of commuting operators on a Hilbert space $K$, and let $D=D_{1} \times \cdots \times D_{N}$ be a polyopen set containing $\sigma(T)$. Then $\bar{D}$ is a complete spectral set for an $N$-tuple $T^{\prime}=\left(T_{1}^{\prime}, \ldots, T_{N}^{\prime}\right)$ of commuting operators similar to $T$.

Proof. For $i=1, \ldots, N$, let $\mu_{i}$ be a measure supported on $\partial \bar{D}_{i}$, such that, if $H_{i}$ is the closure of $\operatorname{Rat}\left(\overline{D_{i}}\right)$ in $L^{2}\left(\mu_{i}\right),\left(D_{i}, H_{i}\right)$ satisfies (1)-(4) in Theorem 1. (This can be arranged by combining Example 3 with Example 4.) Let $\mu$ be the measure $\mu_{1} \times \cdots \times \mu_{N}$ supported on $\partial \bar{D}_{1} \times \cdots \times \partial \bar{D}_{N}$ and let $H$ be the closure of $\operatorname{Rat}(\bar{D})$ in $L^{2}(\mu)$. Then $(D, H)$ satisfies (1)-(4) in the statement of Theorem 2. Since $\sigma(T) \subseteq D$, Theorem 2 implies that $T$ is similar to $T^{\prime}$, where $T^{\prime}$ is the compression of $S_{K}$ to some *-invariant subspace. From this repre- 
sentation, it is clear that $T^{\prime}$ has normal dilation $N=\left(N_{1}, \ldots, N_{N}\right)$ consisting of multiplication by the coordinate functions on $L^{2}(\mu) \otimes K$. Since supp $\mu \subset \partial \bar{D}$, we have $\sigma(N) \subset \partial \bar{D}$, and hence $\bar{D}$ is a complete spectral set for $T^{\prime}$.

The author wishes to thank Professors Robert F. Olin and James E. Thomson for their contributions toward the construction of Example 3.

\section{REFERENCES}

1. M.B. Abrahamse and R.G. Douglas, A class of subnormal operators related to multiply-connected domains, Advances in Math. 19 (1976), 1-43.

2. W. B. Arveson, Subalgebras of C*-algebras. II, Acta Math. 128 (1972), 271-308.

3. C.A. Berger and B.I. Shaw, Intertwining, analytic structure, and the trace norm estimate, Proc. Conf. on Operator Theory (Dalhousie Univ., Halifax, N.S., 1973), edited by P.A. Fillmore, Lecture Notes in Math., vol. 345, Springer-Verlag, Berlin and New York, 1973, pp. 1-12. MR $50 \# 14327$.

4. S. Bergman, The kernel function and conformal mapping, Math. Surveys, no. 5, Amer. Math. Soc., Providence, R.I., 1950. MR 12, 402.

5. J. Bram, Subnormal operators, Duke Math. J. 22 (1955), 75-94. MR 16, 835.

6. J.E. Brennan, Invariant subspaces and rational approximation, J. Functional Analysis 7 (1971), 285-310.

7. D.N. Clark, On commuting contractions, J. Math. Anal. Appl. 32 (1970), 590-596. MR 42 \#2309.

8 R.G.Douglas and C.Pearcy, Invariant subspaces of non-quasitriangular operators, Proc. Conf. on Operator Theory (Dalhousie Univ., Halifax, N.S., 1973), edited by P.A. Fillmore, Lecture Notes in Math., vol. 345, Springer-Verlag, Berlin and New York, 1973, pp. 13-57. MR 50 \# 10857.

9. N. Dunford and J.T. Schwartz, Linear operators. I, General theory, Interscience, New York, 1958. MR 22\#8302.

10. D.N. Herrero, A Rota universal model for operators with mulitply connected spectrum, Rev. Roumaine Math. Pures Appl. 21 (1976), 15-23.

11. G.-C. Rota, On models for linear operators, Comm. Pure Appl. Math. 13 (1960), 469-472. MR $22 \# 2898$.

12. D.E. Sarason, The $H^{p}$ spaces of an annulus, Mem. Amer. Math. Soc. No. 55 (1965). MR $32 \# 6256$.

13. A.L. Shields, Weighted shift operators and analytic function theory, Topics in Operator Theory, Math. Surveys, vol. 13, Amer. Math. Soc., Providence, R.I., 1974. MR 50\# 14341.

14. D. Voiculescu, Norm-limits of algebraic operators, Rev. Roumaine Math. Pures Appl. 19 (1974), 371-378. MR 49 \#7826.

Department of Mathematics, Virginia Polytechnic Institute and State University, BLACKSBURG, VIRGINIA 24061 